\title{
Zitterbewegung in Bogoliubov's System
}

\author{
Yan Li, ${ }^{1}$ Hong-Yi Su, ${ }^{1}$ Fu-Lin Zhang, ${ }^{2,} *$ Jing-Ling Chen, ${ }^{1,}{ }^{3, \dagger}$ Chunfeng Wu ${ }^{4}$ and L. C. Kwek ${ }^{3,5}$ \\ ${ }^{1}$ Theoretical Physics Division, Chern Institute of Mathematics, \\ Nankai University, Tianjin 300071, People's Republic of China \\ ${ }^{2}$ Physics Department, School of Science, Tianjin University, Tianjin 300072, People's Republic of China \\ ${ }^{3}$ Centre for Quantum Technologies, National University of Singapore, 3 Science Drive 2, Singapore 117543 \\ ${ }^{4}$ Pillar of Engineering Product Development, Singapore University \\ of Technology and Design, 20 Dover Drive, Singapore 138682. \\ ${ }^{5}$ National Institute of Education and Institute of Advanced Studies, \\ Nanyang Technological University, 1 Nanyang Walk, Singapore 637616
}

(Dated: March 1, 2018)

\begin{abstract}
We show that Bogoliubov's quasiparticle in superfluid ${ }^{3} \mathrm{He}-\mathrm{B}$ undergoes the Zitterbewegung, as a free relativistic Dirac's electron does. The expectation value of position, as well as spin, of the quasiparticle is obtained and compared with that of the Dirac's electron. In particular, the Zitterbewegung of Bogoliubov's quasiparticle has a frequency approximately $10^{5}$ lower than that of an electron, rendering a more promising experimental observation.
\end{abstract}

PACS numbers: 03.65.Pm, 03.65.-w

\section{INTRODUCTION}

The phenomenon "Zitterbewegung" (ZB), a quivering motion of a free relativistic particle, has drawn many researchers' attention since its theoretical prediction by Schrödinger in 1930 [1]. Nonetheless, the high requirement on measuring precision has ever since defied a direct observation. This triggered proposals [2-6] using some other more experimentally accessible systems to simulate this relativistic quantum effect. For instance, a quantum simulation [7] of the (1+1)-dimensional Dirac's equation using a single trapped ion to mimic the behavior of a free relativistic quantum particle was performed in 2010. In this experiment, the authors studied the ZB for different initial states and measured the particle position as a function of time. The high-level tunability of control in this trapped-ion experiment is also one of the merits.

The origin of ZB of a free particle (usually an electron) within the framework of relativistic quantum mechanics could be attributed to an interference between the positive- and negative-energy components of wave functions. Such understanding is based on the single-particle interpretation of the relativistic quantum mechanics. On the other hand, in the quantum field theory which allows particles (and antiparticles) to be temporarily created and annihilated by satisfying Heisenberg's uncertainty principle, one could interpret the $\mathrm{ZB}$ as the resulting effect of successive scattering between an original electron and virtual electron-positron pairs (i.e. the process of quantum vacuum polarization) [8-10]. Different relativistic quantum theories give distinct explanations of the $\mathrm{ZB}$, be that as it may, it is fairly a reasonable perspective that the $\mathrm{ZB}$ should be present in a great number of

\footnotetext{
*Electronic address: flzhang@tju.edu.cn
}

$\dagger$ Electronic address: chenjl@nankai.edu.cn physical systems [11], possibly including those in which the electron could be in a bound state.

In this Brief Report, we investigate the ZB of a quasiparticle in the superfluid phase of ${ }^{3} \mathrm{He}-B$ [12-16]. Such quasiparticles can be described by Bogoliubov's Hamiltonian, which shares the similar expression of Dirac's Hamiltonian for a free relativistic electron [17][18]. An interesting connectiong between Bogoliubov's Hamiltonian and Dirac's Hamiltonion has been studied in Ref. [19] via braiding relation. We find that the ZB in Bogoliubov's system possesses an amplitude of order $10^{-15} \mathrm{~m}$ and a period of order $10^{-16} \mathrm{~s}$, comparing with an amplitude of order $10^{-12} \mathrm{~m}$ and a period of order $10^{-21} \mathrm{~s}$ for the electron. Thus, the larger period (the lower frequency) of Bogoliubov's quasiparticle renders a promising observation of the $\mathrm{ZB}$ in ${ }^{3} \mathrm{He}-\mathrm{B}$ comparably simpler than that of an electron.

\section{BOGOLIUBOV'S SYSTEM AND ZITTERBEWEGUNG}

The Hamiltonian of Bogoliubov's system for quasiparticles in ${ }^{3} \mathrm{He}-\mathrm{B}$ can be expressed as [17-19]

$$
H_{B}=m(\vec{p}) \beta+c \vec{p} \cdot \vec{\alpha},
$$

where $m(\vec{p})=\vec{p}^{2} / 2 m-\mu, m$ the mass of ${ }^{3} H e, \vec{p}$ the momentum, $\mu$ the chemical potential, $c=\triangle_{B} / k_{F} \hbar$, $\hbar$ the Planck constant, $\triangle_{B}$ the equilibrium order parameter, and $k_{F}$ the Fermi momentum. $\vec{\alpha}=\left(\alpha_{1}, \alpha_{2}, \alpha_{3}\right)$ and $\beta$ are $4 \times 4$ Hermitian matrices satisfying

$$
\begin{aligned}
& \alpha_{i}^{2}=\beta^{2}=1, \\
& \alpha_{i} \beta+\beta \alpha_{i}=0, \\
& \alpha_{i} \alpha_{k}+\alpha_{k} \alpha_{i}=2 \delta_{i k},
\end{aligned}
$$


with $\delta_{i k}=1$ for $i=k$, and $\delta_{i k}=0$ for $i \neq k$. We use the Pauli representation

$$
\vec{\alpha}=\left(\begin{array}{cc}
0 & \vec{\sigma} \\
\vec{\sigma} & 0
\end{array}\right), \beta=\left(\begin{array}{cc}
I & 0 \\
0 & -I
\end{array}\right)
$$

where $I$ is a $2 \times 2$ unit matrix, $\vec{\sigma}=\left(\sigma_{1}, \sigma_{2}, \sigma_{3}\right)$ is a Pauli matrices vector with

$$
\sigma_{1}=\left(\begin{array}{ll}
0 & 1 \\
1 & 0
\end{array}\right), \sigma_{2}=\left(\begin{array}{cc}
0 & -i \\
i & 0
\end{array}\right), \sigma_{3}=\left(\begin{array}{cc}
1 & 0 \\
0 & -1
\end{array}\right) .
$$

This Hamiltonian (1) becomes "relativistic" in the limit $m c^{2} \gg \mu$, where it tends asymptotically toward the Dirac Hamiltonian. However in a real ${ }^{3} \mathrm{He}-\mathrm{B}$, one has an opposite limit $m c^{2} \ll \mu$.

The wave equation of a Bogoliubov's quasiparticle can be written in the form

$$
i \hbar \frac{\partial|\Psi(\vec{x}(t), t)\rangle}{\partial t}=H|\Psi(\vec{x}(t), t)\rangle,
$$

where $|\Psi(\vec{x}(t), t)\rangle$ is a four-component wavefunction. The velocity of the particle is defined and calculated as

$$
\begin{aligned}
\frac{d \vec{x}(t)}{d t} & =\frac{1}{i \hbar}[\vec{x}(t), H] \\
& =\left(\frac{\vec{p}}{m} \beta(t)+c \vec{\alpha}(t)\right) .
\end{aligned}
$$

We should work out $\vec{\alpha}(t)$ first, and it also follows the Heisenberg equation:

$$
\begin{aligned}
\frac{d \vec{\alpha}(t)}{d t} & =\frac{1}{i \hbar}[\vec{\alpha}(t), H] \\
& =\frac{2}{i \hbar}(\vec{\alpha}(t) H-c \vec{p}) .
\end{aligned}
$$

Because the momentum $\vec{p}$ and the Hamiltonian $H$ are constants of motion, this equation can be integrated easily:

$$
\vec{\alpha}(t)=c \vec{p} H^{-1}+\left(\vec{\alpha}(0)-c \vec{p} H^{-1}\right) e^{-2 i H t / \hbar} .
$$

Similarly,

$$
\beta(t)=m(\vec{p}) H^{-1}+\left(\beta(0)-m(\vec{p}) H^{-1}\right) e^{-2 i H t / \hbar} .
$$

Substituting (8) and (9) into Eq. (6), we obtained

$$
\begin{aligned}
& \vec{x}(t)= \vec{x}(0)+\left(c^{2}+\frac{m(\vec{p})}{m}\right) \vec{p} H^{-1} t \\
&+\frac{i \hbar}{2}\left(c \vec{\alpha}(0)+\frac{\vec{p}}{m} \beta(0)-\left(c^{2}+\frac{m(\vec{p})}{m}\right) \vec{p} H^{-1}\right) \\
& \times \frac{e^{-2 i H t / \hbar}-1}{H} .
\end{aligned}
$$

Through this part of the computation, we can see that the operators $\vec{\alpha}$ and $\beta$ depend on time in a nontrivial way. This adds a second term representing a rapidly oscillating motion of the quasiparticles over the conventional velocity operator $\vec{p} H$. This result shows that the quasiparticle exhibits a similar structure as a relativistic electron, and the centroid of the wave-packet

$$
\begin{array}{r}
\mathcal{Z}=\left\langle i \hbar\left(c \vec{\alpha}(0)+\frac{\vec{p}}{m} \beta(0)-\left(c^{2}+\frac{m(p)}{m}\right) \vec{p} H^{-1}\right)\right. \\
\left.\times \frac{e^{-2 i H t / \hbar}-1}{2 H}\right\rangle
\end{array}
$$

represents a rapid oscillatory motion, i.e., the positionZitterbewegung, whose amplitude is of order $v_{F} \frac{\hbar}{2 E} \sim$ $10^{-15} m$ ( with $v_{F}=\frac{\hbar k_{F}}{m}$, and $E$ is the energy), and the period is of order $\frac{\hbar}{E} \sim 10^{-16} s$, respectively.

Energy and Eigenfunction. - Next, we need to obtain the energy and eigenfunction of the Hamiltonian described by the equation (1). It is convenient to express the eigenfunction in the form

$$
|\psi\rangle=\left(\begin{array}{c}
u_{1} \\
u_{2} \\
u_{3} \\
u_{4}
\end{array}\right) e^{i \vec{k} \cdot \vec{x}}=\left(\begin{array}{c}
\phi \\
\varphi
\end{array}\right) e^{i \vec{k} \cdot \vec{x}}
$$

with $\phi=\left(\begin{array}{l}u_{1} \\ u_{2}\end{array}\right)$ and $\varphi=\left(\begin{array}{l}u_{3} \\ u_{4}\end{array}\right)$. Now we define a dichotomous-valued operator

$$
\vec{\Sigma}=\left(\begin{array}{cc}
\vec{\sigma} & 0 \\
0 & \vec{\sigma}
\end{array}\right)
$$

so that

$$
\vec{S}=\frac{\hbar}{2} \vec{\Sigma}
$$

is the spin momentum operator. It is easily to see the set of observables $\{H, \vec{p}, \vec{\Sigma} \cdot \vec{p}\}$ commute with one another. Since

$$
\vec{\Sigma} \cdot \vec{p}|\psi\rangle=\xi|\psi\rangle= \pm \hbar k|\psi\rangle
$$

we have

$$
\begin{aligned}
\hbar \vec{\sigma} \cdot \vec{k} \phi & =\xi \phi, \\
\hbar \vec{\sigma} \cdot \vec{k} \varphi & =\xi \varphi .
\end{aligned}
$$

Here $\phi$ and $\varphi$ are differed by a constant coefficient, so we focus on $\phi$. We have

$$
\left(\begin{array}{cc}
k_{3}-\frac{\xi}{\hbar} & k_{1}-i k_{2} \\
k_{1}+i k_{2} & -k_{3}-\frac{\xi}{\hbar}
\end{array}\right)\left(\begin{array}{c}
u_{1} \\
u_{2}
\end{array}\right)=0
$$

It is easy to get the solutions:

$$
\begin{aligned}
& \text { for } \lambda=+\hbar k, \quad \frac{u_{1}}{u_{2}}=\frac{k_{3}+k}{k_{1}+i k_{2}}=-\frac{k_{1}-i k_{2}}{k_{3}-k}, \\
& \text { for } \lambda=-\hbar k, \quad \frac{u_{1}}{u_{2}}=\frac{k_{3}-k}{k_{1}+i k_{2}}=-\frac{k_{1}-i k_{2}}{k_{3}+k},
\end{aligned}
$$


with $k=|\vec{k}|$.

On the other hand, we substitute (10) into Bogoliubov's Hamiltonian $H$, (i.e. $H \psi=E \psi$ ),

$$
\left(\begin{array}{cc}
m(p)-E & c \vec{\sigma} \cdot \vec{p} \\
c \vec{\sigma} \cdot \vec{p} & -m(p)-E
\end{array}\right)\left(\begin{array}{l}
\phi \\
\varphi
\end{array}\right) e^{i \vec{k} \cdot \vec{r}}=0
$$

Because $\phi, \varphi$ can not all be 0 , i.e.

$$
\left|\begin{array}{cc}
m(p)-E & c \vec{\sigma} \cdot \vec{p} \\
c \vec{\sigma} \cdot \vec{p} & m(p)-E
\end{array}\right|=0,
$$

then we obtain the spectra

$$
E= \pm \sqrt{\eta^{2}+c^{2} \hbar^{2} k^{2}} \equiv E_{ \pm},
$$

with $\eta=\hbar^{2} k^{2} / 2 m-\mu$. Accordingly,

$$
\begin{aligned}
& \text { for } E=E_{+}, \phi=\frac{c \hbar \vec{\sigma} \cdot \vec{k}}{\sqrt{\eta^{2}+c^{2} \hbar^{2} k^{2}}-\eta} \varphi \\
& \text { for } E=E_{-}, \phi=-\frac{c \hbar \vec{\sigma} \cdot \vec{k}}{\sqrt{\eta^{2}+c^{2} \hbar^{2} k^{2}}+\eta} \varphi .
\end{aligned}
$$

From the equations (13) and (14), we know that the eigenvalue of $\vec{\sigma} \cdot \vec{k}$ is $\pm k$. Without explicit normalization, we list all solutions in the following:

(i) when the eigenvalues of $(H, \vec{p}, \vec{\Sigma} \cdot \vec{p})$ are $\left(E_{+}, \hbar \vec{k}, \hbar k\right)$, the eigenfunction is

$$
\left|\psi_{1}\right\rangle=\frac{1}{N_{1}}\left(\begin{array}{c}
k_{1}-i k_{2} \\
k-k_{3} \\
\epsilon_{1}\left(k_{1}-i k_{2}\right) \\
\epsilon_{1}\left(k-k_{3}\right)
\end{array}\right) e^{i \vec{k} \cdot \vec{x}},
$$

where $\epsilon_{1}=\left(\sqrt{\eta^{2}+c^{2} \hbar^{2} k^{2}}-\eta\right) / c \hbar k$.

(ii) when the eigenvalues of $\{H, \vec{p}, \vec{\Sigma} \cdot \vec{p}\}$ are $\left\{E_{+}, \hbar \vec{k},-\hbar k\right\}$, the eigenfunction is

$$
\left|\psi_{2}\right\rangle=\frac{1}{N_{2}}\left(\begin{array}{c}
k_{1}-i k_{2} \\
-k-k_{3} \\
-\epsilon_{2}\left(k_{1}-i k_{2}\right) \\
\epsilon_{2}\left(k+k_{3}\right)
\end{array}\right) e^{i \vec{k} \cdot \vec{x}}
$$

where $\epsilon_{2}=\epsilon_{1}$.

(iii) when the eigenvalues of $\{H, \vec{p}, \vec{\Sigma} \cdot \vec{p}\}$ are $\left\{E_{-}, \hbar \vec{k}, \hbar k\right\}$, the eigenfunction is

$$
\left|\psi_{3}\right\rangle=\frac{1}{N_{3}}\left(\begin{array}{c}
k_{1}-i k_{2} \\
k-k_{3} \\
-\epsilon_{3}\left(k_{1}-i k_{2}\right) \\
-\epsilon_{3}\left(k-k_{3}\right)
\end{array}\right) e^{i \vec{k} \cdot \vec{x}}
$$

where $\epsilon_{3}=\left(\sqrt{\eta^{2}+c^{2} \hbar^{2} k^{2}}+\eta\right) / c \hbar k$.

(iv) when the eigenvalues of $\{H, \vec{p}, \vec{\Sigma} \cdot \vec{p}\}$ are
$\left\{E_{-}, \hbar \vec{k},-\hbar k\right\}$, the eigenfunction is

$$
\left|\psi_{4}\right\rangle=\frac{1}{N_{4}}\left(\begin{array}{c}
k_{1}-i k_{2} \\
-k-k_{3} \\
\epsilon_{4}\left(k_{1}-i k_{2}\right) \\
-\epsilon_{4}\left(k+k_{3}\right)
\end{array}\right) e^{i \vec{k} \cdot \vec{x}},
$$

where $\epsilon_{4}=\epsilon_{3}$.

Position-Zitterbewegung. - To study the ZB, it is necessary to measure $\langle\vec{x}\rangle$, the expectation value of the position operator of the quasiparticles. We introduce the energy projection operators $\Gamma_{ \pm}=\frac{1}{2}(1 \pm \Lambda)$ with $\Lambda=\frac{H}{E_{p}}$ and $E_{p}=\sqrt{\left(\frac{\hbar^{2} k^{2}}{2 m}-\mu\right)^{2}+c^{2} \hbar^{2} k^{2}}$. These operators have the following properties:

$$
\begin{aligned}
& \Gamma_{+}\left|\psi_{+}\right\rangle=\left|\psi_{+}\right\rangle, \\
& \Gamma_{-}\left|\psi_{-}\right\rangle=\left|\psi_{-}\right\rangle, \\
& \Gamma_{+}\left|\psi_{-}\right\rangle=\Gamma_{-}\left|\psi_{+}\right\rangle=0
\end{aligned}
$$

where $\psi_{\lambda}$ is the positive-energy solution and the negativeenergy solution. $\lambda= \pm 1$ is the eigenvalue of the energy projection operators $\Gamma_{ \pm}$. It can be shown that

$$
\begin{aligned}
{\left[\Gamma_{ \pm}, \vec{\alpha}\right] } & = \pm \frac{1}{2 E_{p}}[H, \vec{\alpha}] \\
& = \pm \frac{1}{E_{p}}(c \vec{p}-\alpha H)
\end{aligned}
$$

in additional,

$$
H \Gamma_{ \pm}= \pm E_{ \pm} \Gamma_{ \pm}
$$

Since

$$
\left[\Gamma_{ \pm}, \vec{\alpha}\right]=0
$$

after some simple calculation, we find that

$$
\Gamma_{ \pm}\left(\vec{\alpha}(0)-c \vec{p} H^{-1}\right) \frac{e^{-2 i H t / \hbar}}{2 H} \Gamma_{ \pm}=0 .
$$

Likewise,

$$
\Gamma_{ \pm} \frac{\vec{p}}{m}\left(\beta-m(\vec{k}) H^{-1}\right) \frac{e^{-2 i H t / \hbar}}{2 H} \Gamma_{ \pm}=0 .
$$

Then we have the relationship

$$
\begin{aligned}
\Gamma_{ \pm}\left(c \vec{\alpha}(0)+\frac{\vec{p}}{m} \beta(0)-\right. & \left.\left(c^{2}+\frac{m(\vec{p})}{m}\right) \vec{p} H^{-1}\right) \\
& \times H^{-1} e^{-2 i H t / \hbar} \Gamma_{ \pm}=0 .
\end{aligned}
$$

That is to say, the oscillatory motion vanishes if the wavepacket is a superposition of positive-energy solution only, i.e. is of the form

$$
|\Psi\rangle=\sum \int A(\vec{p}) \psi_{\lambda} d \vec{p}
$$


with $\lambda=+1$, or of negative-energy solutions only $\lambda=$ -1 . It follows that the oscillatory motion is due to interference between the positive- and negative-energy solutions which are normally required to form a wave-packet, since neither set alone constitutes a complete set of functions.

For the superposition state $|\Psi\rangle=\sin \theta\left|\psi_{+}\right\rangle+\cos \theta\left|\psi_{-}\right\rangle$, one may calculate the position-Zitterbewegung as

$$
\mathcal{Z}=\frac{m(k) \hbar^{2} k^{2}-E_{+}^{2}}{E_{+}^{2}} \frac{c \hbar}{m k E_{+}} \vec{k} \sin 2 \theta \sin \frac{2 E_{+} t}{\hbar}
$$

for $|\Psi\rangle=\sin \theta\left|\psi_{+}\right\rangle+\cos \theta\left|\psi_{-}\right\rangle$. This is the expectation value of oscillatory motion, that is an interference effect between the positive and negative-energy parts. It does not appear in the case that spinors consist entirely of positive-energy ( negative-energy) parts.

Spin-Zitterbewegung. - Moreover, we have a look at the spin of the Bogoliubov's system, and see whether it has the same properties as the ZB. The spin operator satisfies the Heisenberg equation

$$
\begin{aligned}
\frac{d \vec{S}}{d t}(t) & =\frac{1}{i \hbar}[\vec{S}(t), H] \\
& =-c \vec{\alpha}(0) \times \vec{p} e^{-2 i H t / \hbar}
\end{aligned}
$$

which can be integrated easily, so that

$$
\left.\vec{S}(t)=\vec{S}(0)-\frac{i \hbar}{2}(c \vec{\alpha}(0) \times \vec{p}) H^{-1}\left(e^{-2 i H t / \hbar}-1\right) 30\right)
$$

There has a rapid oscillatory motion, i.e., spinZitterbewegung as

$$
\mathcal{Z}_{\text {spin }}=\left\langle\frac{i \hbar}{2}(c \vec{\alpha}(0) \times \vec{p}) \frac{e^{-2 i H t / \hbar}}{H}\right\rangle .
$$

Similar to the above analysis, we observe that the expectation values of spin for some types of initial superpositions of positive- and negative-energy wavefunctions strangely vanish. That is, $\left\langle\psi_{i}|\vec{S}| \psi_{i}\right\rangle=0,\left\langle\psi_{1}|\vec{S}| \psi_{2}\right\rangle=$ $0,\left\langle\psi_{1}|\vec{S}| \psi_{3}\right\rangle=0,\left\langle\psi_{2}|\vec{S}| \psi_{4}\right\rangle=0,\left\langle\psi_{1}|\vec{S}| \psi_{4}\right\rangle \neq$ $0,\left\langle\psi_{2}|\vec{S}| \psi_{3}\right\rangle \neq 0$

\section{CONCLUSION}

To summarize, we have discussed the position-ZB in Bogoliubov's system and shown that, besides classical uniform motion, the centroid of the wave-packet has a rapid oscillatory motion. The expectation value of the rapid oscillatory motion has been obtained, indicating an interference between the positive- and negative-energy wavefunctions The ZB of Bogoliubov's quasiparticle has a frequency dramatically lower than that of a free Dirac's electron, rendering a promising observation comparably simpler. We have also discussed the spin-ZB in Bogoliubov's system in the end.

We thank E. Solano for valuable discussion. F.L.Z. is supported by NSF of China (Grant No. 11105097). J.L.C. is supported by National Basic Research Program (973 Program) of China under Grant No. 2012CB921900, NSF of China (Grant Nos. 10975075 and 11175089) and also partly supported by National Research Foundation and Ministry of Education, Singapore.
[1] E. Schröding, Sitz. Preuss. Akad. Wiss. Phys.-Math. KI. 24, 418 (1930).

[2] J. Schliemann, D. Loss, and R. M. Westervelt, Phy. Rev. Lett. 94, 206801 (2005).

[3] L. Lamata, J. León, T. Schätz, and E. Solano, Phy. Rev. Lett. 98, 253005 (2007).

[4] A. Bermudez, M. A. Martin-Delgado, and E. Solano, Phy. Rev. A 76, 041801(R) (2007).

[5] X. Zhang, Phy. Rev. Lett. 100, 113903 (2008).

[6] J. Y. Vaishnav and C. W. Clark, Phy. Rev. Lett. 100, 153002 (2008).

[7] R. Gerritsma, G. Kirchmair, F. Zähringer, E. Solano, R. Blatt, and C. F. Roos, Nature 643, 68(2010).

[8] P. Krekora, Q. Su, and R. Grobe, Phy. Rev. Lett. 93, 043004 (2004).

[9] J. Schliemann, D. Loss, and R. M. Westervelt, Phy. Rev. Lett. 94, 206801 (2005).

[10] Z. Y. Wang, C. D. Xiong, Phy. Rev. A 77, 045402 (2008).

[11] D. Lurié, and S. Cremer, Physica 50, 224-240 (1970).
[12] Y. M. Bunkov, S. N. Fisher, A. M. Guénault, C. J. Kennedy, and G. R. Pickett, Phy. Rev. Lett. 68, 5 (1992).

[13] P. J. Hakonen, M. Krusius, M. M. Salomaa, and J. T. Simola, Phys. Rev. Lett. 51, 1362 (1983).

[14] T. D. C. Bevan, A. J. Manninen, J. B. Cook, A. J. Armstrong, J. R. Hook, and H. E. Hall, Phys. Rev. Lett. 74, 750 (1995).

[15] S. N. Fisher, A. J. Hale, A. M. Gunault, and G. R. Pickett, Phys. Rev. Lett. 86, 244 (2001).

[16] D. I. Bradley, D. O. Clubb, S. N. Fisher, A. M. Guénault, R. P. Haley, C. J. Matthews, G. R. Pickett, V. Tsepelin, and K. Zaki, Phys. Rev. Lett. 96, 035301 (2006).

[17] G. E. Volovik, Exotic Properties of Superfluid ${ }^{3} \mathrm{He}-\mathrm{B}$ (World Scientific, Singapore, 1992), p. 77.

[18] Volovik, E. Grigorij, Physics Reports 35(4), 195-348 (2001).

[19] B. X. Xie, K. Xue, and M. L. Ge, Phy. Rev. A 77, 064101 (2008). 